\title{
ON INVARIANT CONNECTIONS OVER A PRINCIPAL FIBRE BUNDLE*
}

\author{
HSIEN-CHUNG WANG
}

\section{Introduction}

The invariant affine connection over a coset space $G / J$ of a Lie group $G$ have been discussed by various authors. Recently. Nomizu [8] gave a systematic study of this problem when $J$ is reductible in $G$. Among other results, he established a 1-1 correspondence between the invariant affine connections and certain multilinear mappings, and calculated the torsion and curvature. For canonical affine connection of the second kind, the holonomy group was also given.

It is the purpose of this paper to discuss the connections over a principal fibre bundle which admit a fibre transitive ${ }^{1)}$ Lie group of automorphisms without restricting to the reductible case. In fact, let $\{E, S\}$ be a differentiable principal fibre bundle with total space $E$, structural group $S$, and base space $B$. Suppose $G$ to be a Lie group of automorphisms of $\{E, S\}$, and $J$ the subgroup leaving a fibre $F_{0}$ invariant. There is a natural homomorphism $\psi: J \rightarrow S$. If we regard $G$ as a transformation group of the base space $B$, then $J$ is the isotropic subgroup at the point $b_{0} \in B$ which corresponds to $F_{0}$. (In the particular case that $E$ is the bundle of frames of $B$, then $\psi$ is nothing but the linear representation of $J$ on the tangent space of $B$ at $b_{i}$.) Let us denote by $\hat{\mathbf{G}}, \hat{\mathbf{S}}, \hat{\mathbf{J}}$ the Lie algebras of $G, S, J$ respectively. The main results can be stated as follows:

(A) Suppose $G$ to be iransitive on the fibres of $E$. Then there is a 1-1 correspondence between the G-invariant connections over $E$ and the linear mappings $\Psi: \hat{\mathbf{G}} \rightarrow \hat{\mathbf{S}}$ such that (1) $\Psi \circ \operatorname{Ad} . j=\operatorname{Ad} . \psi(j) \circ \Psi, j \in J$, and $(2) \Psi(\vec{j})$ $=\psi(\vec{j}), \vec{j} \in \hat{\mathfrak{j}}$ where we use $\psi$ to denote both the group homomorphism: $J \rightarrow S$ mentioned above and the Lie algebra homomorphism: $\hat{\mathbf{J}} \rightarrow \hat{\mathbf{S}}$ it induces.

Received October 18, 1957.

Revised February 19, 1958.

* Sponsored by National Science Foundation.

1) By fibre transitive, we mean that, given any two fibres, there exists an element of the group carrying one to the other. 
(B) Suppose, moreover, that E satisfies the second countability axiom. Let $\hat{\mathbf{V}}$ denote the linear subspace of $\hat{\mathbf{S}}$ spanned by all the vectors of the form $\Psi\left[\vec{g}_{1}, \vec{g}_{2}\right]-\left[\Psi \vec{g}_{1}, \Psi \vec{g}_{2}\right], \vec{g}_{1}, \vec{g}_{2} \in \hat{\mathbf{G}}$. Then the Lie algebra of the holonomy group of the connection which corresponds to $\Psi$ is given by

$$
\hat{\mathbf{V}}[\Psi(\hat{\mathbf{G}}), \hat{\mathbf{V}}]+[\Psi(\hat{\mathbf{G}}),[\Psi(\hat{\mathbf{G}}), \hat{\mathbf{V}}]]+\ldots
$$

In the discussion of the holonomy group of a $G$-invariant connection ( $G$ not necessarily fibre-transitive), we introduce a subgroup $\Delta$ of $S$ which depends on $G$. The group $\Delta$ contains both the holonomy group and the group $\psi(J)$. Elements of $\Delta$ was first used by Nomizu [9], and the Lie algebra of $\Delta$ is closely related with a tensor studied by Kostant [12] when $\{E, S\}$ is the bundle of frames of a manifold. Interpreting some known results in terms of $\Delta$, we get the following:

Let $G$ be a connected group of isometries of a compact Riemannian manifold $B$. Then, at each point of $B$, the linear isotropic subgroup is contained in the holonomy group. Moreover, each parallel tensor field over $B$ is invariant under $G$.

When $B$ is homogeneous, this has been proved by B. Kostant [13].

\section{Some conventions}

Throughout this paper, all differentiable menifolds and mappings are understood to be of class $C^{\infty}$.

Let $E$ be a differentiable manifold and $x$ a point of $E$. We shall always use $T(E)$ to denote the tangent bundle of $E$, and $T_{x}(E)$ the tangent space of $E$ at $x$. Suppose $f: E \rightarrow M$ to be a differentiable mapping of $E$ into a differentiable manifold $M$. Then $f$ induces a mapping $f^{\prime}: T(E) \rightarrow T(M)$ which is called the differential of $f$. Restricted to $T_{x}(E), f^{\prime}$ is linear. Let $V$ be a vector space over the reals. We denote by $\Theta_{E}$ and $\Theta_{k}$, respectively, the sets of linear combinations of $V$-valued differential forms over $E$ and over $M$. They are graded algebras with differential operator. The dual of $f^{\prime}$ gives a homomorphism $f^{4}: \Theta_{M} \rightarrow \Theta_{E}$.

For the sake of simplicity, we shall use, in what follows, the same symbol $f$ to denote the differential $f^{\prime}$ of a differentiable mapping $f$.

Let $G$ be a Lie group and $\hat{\mathbf{G}}$ its (left) Lie algebra. We identify $\hat{\mathbf{G}}$ with the tangent space $T_{c}(G)$ of $G$ at the identity $e$ of $G$. For $g \in G, L_{g}$ and $R_{g}$ denote, respectively, the left and right translations induced by $g$. The composite 
$L_{g} \circ R_{g-1}$ is called the adjoint transformation and is denoted by Ad. $g$. It carries $T_{e}(G)$ into itself and is an automorphism of the Lie algebra of $G$. By left Maurer-Cartan form, we mean the $\hat{\mathbf{G}}$-valued linear differential form $\omega$ over $G$ such that $\omega(\vec{g})=\left(L_{g^{-1}}\right)(\vec{g})$ for $\vec{g} \in T_{g}(G)$. Right Maurer-Cartan form is defined in the similar manner.

Let $\psi$ be a linear representation of a Lie group $G$ on a linear space $V$, and $H$ a closed subgroup of $G$. Suppose $\lambda: T_{e}(G) \rightarrow V$ to be a linear mapping such that $\lambda \circ$ Ad. $h=\varphi(h) \circ \lambda$ for all $h \in H$. We can extend $\lambda$ to a $V$-valued linear differential form $\widetilde{\omega}$ over $G$ by putting

$$
\widetilde{\omega}(\vec{g})=\varphi(g) \lambda\left(\left(L_{g^{-1}}\right)(\vec{g})\right), \quad \vec{g} \in T_{g}(G), \quad g \in G .
$$

Its differentiability follows from the differentiability of the group multiplication of $G$. For any $k \in G$, and $h \in H$, we find by a direct calculation that

$$
\left(L_{k}\right)^{*} \widetilde{\omega}=\varphi(k) \bar{\omega}, \quad(\text { Ad. } h)^{*} \widetilde{\omega}=\varphi(h) \widetilde{\omega} .
$$

This $\widetilde{\omega}$ will be called the $\varphi$-invariant extension of $\lambda$. We note that $\widetilde{\omega}$ is the only $V$-valued differential form which extends $\lambda$ and satisfies the first part of the conditions (2.1).

\section{3. ç-invariant differential forms}

Suppose that a Lie group $G$ acts on a differentiable manifold $E$ differentiably. Then the mapping $G \times E \rightarrow E$ given by $(g, x) \rightarrow g(x), g \in G, x \in E$, is differentiable $\left[7\right.$, p. 212]. Choose a point $x_{0}$ of $E$, and define $p: G \rightarrow E$ by $p(g)=g\left(x_{0}\right)$. This mapping $p$ is differentiable and the subgroup $H=\{g: g \in G$, $\left.g\left(x_{0}\right)=x_{0}\right\}$ is a closed subgroup of $G$. For any $g \in G$, and $h \in H$, we find immediately that the diagrams
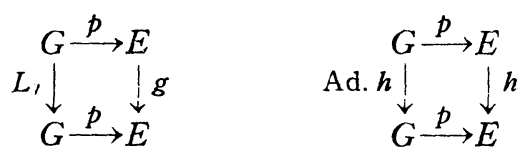

are commutative, and hence

$$
\left(L_{g}\right)^{*} p^{*}=p^{*} g^{*}, \quad(\text { Ad. } h)^{*} p^{*}=p^{*} h^{*} .
$$

Let $\varphi$ be a linear representation of $G$ over a linear space $V$. A $V$-valued differential form $\omega$ over $E$ is called $\varphi$-invariant under $G$ if $g^{*} \omega=\varphi(g) \omega$ for all $g$ of $G$. 
(3.3) Let $\widetilde{\omega}=p^{*} \omega$ where $\omega$ is a c-invariant, V-valued linear difjerential form over $E$. Then (i) $L_{g}^{*} \widetilde{\omega}=c ̧(g) \widetilde{\omega},(\text { Ad. } h)^{*} \widetilde{\omega}=c(h) \widetilde{\omega}$ for $g \in G, h \in H$, and (ii) $\widetilde{a}(\vec{h})=0$ for $\vec{h} \in T_{e}(H)$.

Proof. Property (i) follows directly from (3.2). To see property (ii), we note that $p(H)=x_{0}$. Hence, for $\vec{h} \in T_{e}(H), p(\vec{h})=0$ and

$$
\widetilde{\omega}(\vec{h})=\left(p^{*} \omega\right)(\vec{h})=\omega(p(\vec{h}))=0 .
$$

(3.4) Suppose that $G$ acts on $E$ transitively, and that $\widehat{\omega}$ is a V-valued, linear form over $G$ having the properties (i) and (ii) in (3.3). There exists then a unique $\varphi$-invariant, $V$-valued differential from $\omega$ over $E$ such that $\widetilde{\omega}=p^{*} \omega$.

Proof. The uniqueness follows from the fact that $p$ is an onto mapping. To see the existence, let us consider the restriction of $p$ on $T_{e}(G)$. This is a linear mapping: $T_{e}(G) \rightarrow T_{x_{0}}(E)$. From the theory of Lie groups, we know that this mapping is onto and has $T_{e}(H)$ as its kernel. Let $\widetilde{\omega}_{e}$ denote the restriction of $\widetilde{\omega}$ on $T_{e}(G)$. Since $\widetilde{\omega}_{e}$ vanishes in $T_{e}(H)$, there exists a linear mapping $\lambda: T_{x_{0}}(E) \rightarrow V$ such that

$$
\widetilde{\omega}_{e}(\vec{k})=\lambda(p(\vec{k})), \quad \vec{k} \in T_{e}(G) .
$$

For any $\vec{x} \in T_{x_{0}}(E)$, we choose $\vec{k} \in T_{e}(G)$ with the property that $p(\vec{k})=\vec{x}$. From the commutativity of the diagrams (3.1) and the property (i) of $\widetilde{\omega}$, it follows that

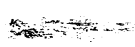

$$
\begin{gathered}
\lambda(h(\vec{x}))=\lambda\left(h(p(\vec{k}))=\lambda(p(\operatorname{Ad} . h)(\vec{k}))=\widetilde{\omega}_{e}((\operatorname{Ad} . h)(\vec{k}))\right. \\
\left((\operatorname{Ad} . h)^{*} \widetilde{\omega}\right)(\vec{k})=\varphi(h) \tilde{\omega}(\vec{k})=\varphi(h) \lambda(\vec{x}), \quad h \in H .
\end{gathered}
$$

Let $y$ be any point of $E$, and $\vec{y} \in T_{y}(E)$. Since $G$ is transitive on $E$, there exists an element $g$ of $G$ such that $g(y)=x_{j}$. Hence $g(\vec{y}) \in T_{x_{0}}(E)$, and $\lambda(g(\vec{y}))$ has a meaning. We shall see that the expression $\varphi\left(g^{-1}\right) \lambda(g(\vec{y}))$ is independent of the choice of $g$. Suppose that $g_{1}$ also carries $y$ to $x_{j}$. Then $g_{1}=h g$ for a certain $h$ of $H$. From (3.6), we have

$$
\varphi\left(g_{1}^{-1}\right) \lambda\left(g_{1}(\vec{y})\right)=\varphi\left(g^{-1}\right) \varphi\left(h^{-1}\right) \lambda\left(h(g(\vec{y}))=\varphi\left(h^{-1}\right) \lambda(g(\vec{y})) .\right.
$$

Thus $\varphi\left(g^{-1}\right) \lambda(g(\vec{y}))$ depends only on $\vec{y}$. Defining $\omega(\vec{y})=\varphi\left(g^{-1}\right) \lambda(g(\vec{y}))$, we get a $V$-valued linear form $\omega$ over $E$. Its' differentiability follows from the differentiability of the transformation mapping: $G \times E \rightarrow E$, while its $\varphi$-invari- 
ance under $G$ follows from its definition. Furthermore, we can easily verift that $\widetilde{\omega}=p^{*} \omega$ by using (3.5), the $\varphi$-invariance of $\omega$ and the property (i) of $\widetilde{\omega}$.

\section{Automorphisms of a principal fibre space}

Let $S$ be a topological transformation group of a space $E$ acting from the left. The system $\{E, S\}$ is said to form a principal fibre space if the following two conditions are satisfied:

1) Given any two points $x, y$ of $E$, there exists at most one element of $S$ carrying $x$ to $y$.

2) The set $R=\{(x, y)$ : there exists $s$ of $S$ carrying $x$ into $y\}$ is closed in $E \times E$, and the mapping of $R$ into $S$ defined by $(x, y) \rightarrow s$ is continuous.

The group $S$, the orbits under $S$, and the space $B$ of orbits are called, respectively, the structure group, fibres, and the base space. From condition 2 ), it follows that fibres are closed in $E$ and are all homeomorphic with $S$. Here, contrary to convention, we assume the group $S$ to act on $E$ from the left. This has some advantages in our further discussions.

A principal fibre space will be called differentiable if all the spaces involved in the definition are differentiable manifolds and all the mappings are differentiable.

Let $\{E, S\}$ be a differentiable principal fibre space. Then $S$ is a Lie group acting on $E$ differentiably. From Frobenius Theorem, we know that local differentiable cross section exists. Then the base space $B$ has a natural differentiable structure, and $E$ is a differentiable fibre bundle over $B$. A transformation of $E$ is called an automorphism if it is bi-differentiable and it permutes with every element of $S$. Hence an automorphism carries fibres into fibres. Now suppose $G$ to be a Lie group of automorphisms of $E$. Choose a fibre $F_{0}$ of $E$ and a point $x_{0}$ in $F_{0}$. Consider the subgroup $J=\left\{g: g \in G, g\left(F_{0}\right)=F_{0}\right\}$. For $j \in J$, $j^{-1}\left(x_{0}\right) \in F_{0}$. There exists then a unique element, say $\psi(j)$, of $S$ such that $\psi(j)\left(x_{0}\right)=j^{-1}\left(x_{0}\right)$. Since elements of $J$ and elements of $S$ permute, the mapping $\psi: J \rightarrow S$ preserves the group multiplication. It is, moreover, continuous on account of the property 2 ) in the definition of a principal fibre space. We note that for different choice of the point $x_{0}$, the homomorphisms obtained differ only by an inner automorphism of $S$.

Let $Q=G \times S$ be the direct product of $G$ and $S$. In the natural manner, 
$Q$ acts on $E$ as a differentiable transformation group $(Q$ is not necessarily effective even if both $G$ and $S$ are so). The isotropic subgroup of $Q$ at $x_{0}$ is then $H=\{(j, \phi(j)): j \in J\}$. Let $\hat{\mathbf{J}}, \hat{\mathbf{S}}, \hat{\mathbf{H}}$ denote the Lie algebras of $J, S, H$ respectively. Following our convention, we use the same letter $\psi$ to denote the algebra homomorphism: $\hat{\mathbf{J}} \rightarrow \hat{\mathbf{S}}$ induced by the group homomorphism $\psi$. Then

$$
\hat{\mathbf{H}}=\{\vec{j}+\psi(\vec{j}): \vec{j} \in \hat{\mathbf{J}}\} .
$$

In particular, suppose $E$ to be the bundle of frames of a differentiable manifold $B$ of dimension $n$. Then the structure group $S$ is the general real linear group $G L(R, n)$. Let $G$ be a differentiable transformation group of $B$, and $J^{\prime}$ the isotropic subgroup at a point $b$ of $B$. Each $j$ of $J^{\prime}$ induces a linear transformation $\tilde{j}$ of the tangent space $T_{b}(B)$. Thus we have a homomorphism $j \rightarrow \tilde{j}$ of $J$ into $G L(R, n)$. On the other hand, $G$ can be regarded as a group of automorphisms of the bundle $E$ of frames. Let $F_{0}$ be the fibre of $E$ corresponding to $b$, and $J, \psi$ have the same meaning as in the preceding paragraph. Then we see readily that $f=J^{\prime}$ and that $\psi$ is nothing but the linear representation $j \rightarrow \tilde{j}$.

\section{Invariant connections}

Let $\{E, S\}$ be a differentiable principal fibre space, and $\hat{\mathbf{S}}$ the Lie algebra of $S$. A connection over $E$ is, by definition [2, p. 51], an $\hat{\mathbf{S}}$-valued linear differential form $\omega$ over $E$ such that (a) $s^{*} \omega=($ Ad. $s) \omega, s \in S$; and (b) if $y$ is any point of $E$ and $\pi: S \rightarrow E$ is defined by $\pi(s)=s(y)$, then $\pi^{*} \omega$ is the right MaurerCartan form of $S$. Here, we have Ad. $s$ and right Maurer-Cartan form instead of Ad. $s^{-1}$ and left Maurer-Cartan form as in the classical definition. This is due to our assumption that $S$ acts on $E$ from the left.

Let $\omega$ be a connection over $E$, and $G$ a Lie group of automorphisms of $E$ leaving $\omega$ invariant. Consider the direct product $Q=G \times S$, and denote by $\varphi$ the representation of $Q$ on $\hat{\mathbf{S}}$ defined by $\varphi(g, s)=$ Ad. $s, g \in G, s \in S$. Then the form $\omega$ is $\varphi$-invariant under $Q$. Choose a fibre $F_{0}$ of $E$, and a point $x_{0}$ in $F_{0}$. Let

$$
J=\left\{g: g \in G, g\left(F_{0}\right)=F_{0}\right\}, \quad H=\left\{q: q \in Q, q\left(x_{0}\right)=x_{0}\right\},
$$

and $p: Q \rightarrow E$ be defined by $p(q)=q\left(x_{0}\right)$. 
(5.1) Denote by $\Psi$ the restriction of $-p^{*} \omega$ on $T_{e}(G)$. Then

$$
\Psi \circ(\text { Ad. } j)=\operatorname{Ad} . \varphi(j) \circ \Psi, \quad \Psi(\vec{j})=\psi(\vec{j})
$$

for all $j \in J$ and $\vec{j} \in \hat{\mathbf{J}}$, where $\psi$ is the homomorphism discussed in $\S 4$.

Proof. Since $\omega$ is $\varphi$-invariant under $Q$, we have from (3.3)

$$
(\text { Ad. } h)^{*}\left(p^{*} \omega\right)=\varphi(h)\left(p^{*} \omega\right), \quad\left(p^{*} \omega\right)(\vec{h})=0, \quad h \in H, \quad \vec{h} \in \hat{\mathbf{H}} .
$$

Let $\vec{g} \in \hat{\mathbf{G}}=T_{e}(G)$, and $j \in J$. The element $h=(j, \phi(j))$ belongs to $H$. (5.2) then implies that

$$
\Psi((\operatorname{Ad} . h)(\vec{g}))=\varphi(h) \Psi(\vec{g})=\operatorname{Ad} . \psi(j) \Psi(\vec{g}) .
$$

From the permutability of elements of $G$ and $S$, we know (Ad. $h)(\vec{g})=($ Ad. $j)(\vec{g})$. Hence $\Psi(($ Ad. $j)(\vec{g}))=$ Ad. $\psi(j) \Psi(\vec{g})$.

Let $\vec{j} \in \hat{\mathbf{J}}$. From (4.1), $\vec{j}+\psi(\vec{j}) \in T_{e}(H)$, and hence by (5.2), we have $\left(p^{*} \omega\right)(\vec{j}+\psi(\vec{j}))=0$. In other words, $\Psi(\vec{j})=\left(p^{*} \omega\right)(\psi(\vec{j}))$. By definition of a connection, the restriction of $p^{*} \omega$ on $S$ is the right Maurer-Cartan form of $S$. From the fact $\psi(\vec{j}) \in T_{e}(S)$, it follows then that $\left(p^{*} \omega\right)(\psi(\vec{j}))=\psi(\vec{j})$. Therefore, $\Psi(\vec{j})=\psi(\vec{j})$ for $\vec{j} \in \hat{\mathbf{j}}$. Proposition (5.1) is thus proved.

(5.2) Let $\Omega$ be the curvature form of a connection $\omega$ over $E$ invariant under $G$. Then the restriction of $p^{*} \Omega$ in $T_{e}(G)$ is given by

$$
2\left(p^{*} \Omega\right)\left(\vec{g}_{1}, \vec{g}_{2}\right)=\Psi\left[\vec{g}_{1}, \vec{g}_{2}\right]-\left[\Psi\left(\vec{g}_{1}\right), \Psi\left(\vec{g}_{2}\right)\right], \quad \vec{g}_{1}, \vec{g}_{2} \in T_{e}(G) .
$$

Proof. From equations of Cartan, we know that

$$
2 \Omega=2 d \omega+[\omega, \omega] .
$$

It follows that $2 p^{*} \Omega=2 d\left(p^{*} \omega\right)+\left[p^{*} \omega, p^{*} \omega\right]$, and hence

$$
2\left(p^{*} \Omega\right)\left(\vec{g}_{1}, \vec{g}_{2}\right)=2 d\left(p^{*} \omega\right)\left(\vec{g}_{1}, \vec{g}_{2}\right)+\left[\Psi\left(\vec{g}_{1}\right), \Psi(\vec{g})\right], \quad \vec{g}_{1}, \vec{g}_{2} \in T_{e}(G) .
$$

The linear form $\omega$ over $E$ is $\varphi$-invariant. We have therefore, from (3.3) that $L_{g}^{*}\left(p^{*} \omega\right)=\varphi(g)\left(p^{*} \omega\right)=p^{*} \omega$ for every $g$ of $G$. In other words, the restriction of $p^{*} \omega$ on $G$ is invariant under left translations. Hence

$$
2 d\left(p^{*} \omega\right)\left(\vec{g}_{1}, \vec{g}_{2}\right)+\left(p^{*} \omega\right)\left[\vec{g}_{1}, \vec{g}_{2}\right]=0
$$

and hence

$$
2\left(p^{*} \Omega\right)\left(\vec{g}_{1}, \vec{g}_{2}\right)=\left[\Psi\left(\vec{g}_{1}\right), \Psi\left(\vec{g}_{2}\right)\right]-\Psi\left[\vec{g}_{1}, \vec{g}_{2}\right]
$$




\section{Fibre-transitive case}

Suppose that $E, S, G, x_{0}, J, \varphi, \ldots$ have the same meaning as in the preceding section. We see immediately that the following three conditions are equivalent: (i) $G$ is transitive on the fibres of $E$; (ii) the group $Q=G \times S$ is transitive on $E$; and (iii) $G$, when regarded as a transformation group of the base space $B$, is transitive. Moreover, if this is the case, then $B$ is homeomorphic with the coset space $G / J$.

THEOREM 1. Let $G$ be a Lie group of automorphisms of a differentiable principal fibre space $\{E, S\}$, transitive on the fibres. Then there is a one-to-one correspondence between the invariant connections $\omega$ over $E$ and the linear mappings $\Psi: \hat{\mathbf{G}} \rightarrow \hat{\mathbf{S}}$ such that

$$
\Psi \circ(\text { Ad. } j)=\operatorname{Ad} . \psi(j) \circ \Psi, \quad \Psi(\vec{j})=\psi(\vec{j}), \quad j \in J, \quad \vec{j} \in \hat{\mathbf{J}} .
$$

Let $\Omega$ denote the curvature form of $\omega$. Then the restriction of $p^{*} \Omega$ on $G$ is given by

$$
2\left(p^{*} \Omega\right)\left(\vec{g}_{1}, \vec{g}_{2}\right)=\left[\Psi\left(\vec{g}_{1}\right), \Psi\left(\vec{g}_{2}\right)\right]-\Psi\left[\vec{g}_{1}, \vec{g}_{2}\right], \vec{g}_{1}, \vec{g}_{2} \in \hat{\mathbf{G}} .
$$

Proof. On account of (5.1) and (5.2), it suffices to prove that to a linear mapping $\Psi: \hat{\mathbf{G}} \rightarrow \hat{\mathbf{S}}$ with the two properties mentioned in the Theorem, there corresponds an invariant connection $\omega$ under $G$. For this purpose, we extend $-\Psi$ to a linear mapping $\lambda: T_{e}(Q) \rightarrow \hat{\mathbf{S}}$ by putting

$$
\lambda(\vec{g}+\vec{s})=\vec{s}-\Psi(\vec{g}), \quad \vec{g} \in \hat{\mathbf{G}}, \quad \vec{s} \in \hat{\mathbf{S}} .
$$

Let $h$ be an element of $H$. There exists $j \in J$ such that $h=(j, \psi(j))$. Taking account of the fact that $G$ and $S$ are elementwise permutable, we have

$$
\begin{aligned}
\lambda((\operatorname{Ad} . h)(\vec{g}+\vec{s})) & =\lambda((\operatorname{Ad} . j)(\vec{g}))+((\operatorname{Ad} . \psi(j))(\vec{s})) \\
& =\operatorname{Ad} . \psi(j)(\vec{s})-\Psi((\operatorname{Ad} . j)(\vec{g})) \\
& =\operatorname{Ad} \phi(j)(\vec{s})-(\operatorname{Ad} . \psi(j)) \Psi(\vec{g})=\operatorname{Ad} . \psi(j) \lambda(\vec{g}+\vec{s}) .
\end{aligned}
$$

In other words, $\lambda \circ$ Ad. $h=\varphi(h) \circ \lambda$ for $h \in H$. Therefore $\lambda$ has a $\varphi$-invariant extension $\widetilde{\omega}$ over the entire tangent space $T(Q)$ of $Q$ as defined is $\S 2$. This $\widetilde{\omega}$ is an $\hat{\mathbf{S}}$-valued linear differential form over $Q$ with the following properties:

2) The Lie algebra $\hat{\mathbf{G}}$ is always identified with $\mathrm{T}_{e}(G)$. 


$$
\begin{gathered}
L_{l}^{*} \widetilde{\omega}=\varphi(q) \widetilde{\omega}, \quad(\text { Ad. } h)^{*} \widetilde{\omega}=\varphi(h) \widetilde{\omega}, \quad \widetilde{\omega}(\vec{s})=\vec{s}, \quad \widetilde{\omega}(\vec{g})=-\Psi(\vec{g}) \\
q \in Q, \quad h \in H, \quad \vec{g} \in \hat{\mathbf{G}}, \quad \vec{s} \in \hat{\mathbf{S}} .
\end{gathered}
$$

Let $\vec{h} \in T_{e}(H)=\hat{\mathbf{H}}$. From (4.1), we can find $\vec{j} \in \hat{\mathbf{J}}$ such that $\vec{h}=\vec{j}+\psi(\vec{j})$. Then

$$
\widetilde{\omega}(\vec{h})=\psi(\vec{j})-\Psi(\vec{j})=0 .
$$

Taking account of (6.1) and (6.2), we have from (3.4) that there exists an $\hat{S}$-valued linear form $\omega$ over $E$ which is $\varphi$-invariant under $Q$ and satisfies $\widetilde{\omega}=p^{*} \omega$. We shall see that this $\omega$ is a connection over $E$ invariant under the group $G$.

For $g \in G, s \in S$, we have from the definition of $\&$ that $\varphi(g)=$ identity, and $\psi(s)=$ Ad. $s$. Therefore, the $\varphi$-invariance of $\omega$ under $Q$ accounts to

$$
g^{*} \omega=\omega, \quad s^{*} \omega=(\text { Ad. } s) \omega .
$$

Let $\theta$ be the restriction of $\widetilde{\omega}$ on $S$. From (6.1), we have

$$
L_{s}^{*} \theta=(\text { Ad. } s) \theta, \quad \theta(\vec{s})=\vec{s}, \quad s \in S, \quad \vec{s} \in \hat{\mathbf{s}} .
$$

These two equalities imply that $\theta$ is the right Maurer-Cartan form of $S$. Now let $y$ be any point of $E$, and $\pi: S \rightarrow E$ be defined by $\pi(s)=s(y), s \in S$. Since $G$ is transitive on the fibres of $E, Q$ is transitive on $E$. There exist $g_{0}$ of $G$ and $s_{0}$ of $S$ such that $g_{0}^{-1} s_{0}\left(x_{0}\right)=y$. From the commutativity of the diagram

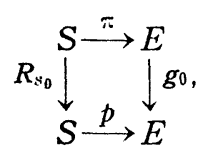

it follows that $\pi^{*} \omega=\pi^{*} g_{0}^{*} \omega=S_{s_{0}}^{*} p^{*} \omega=R_{s_{0}}^{*} \theta=0$, and hence $\pi^{*} \omega$ is the right Maurer-Cartan form of $S$. Combining this with (6.8), we know that $\omega$ is a connection over $E$ invariant under $G$. Theorem $I$ is thus proved.

Corollary 1. In Theorem 1, the connection $\omega$ is locally flat if and only if the linear mapping $\Psi: \hat{\mathbf{G}} \rightarrow \hat{\mathbf{S}}$ is a Lie algebra homomorphism.

Proof. The "only if" part is a direct consequence of Theorem 1. To see the "if" part, let us assume that $\Psi$ is a Lie algebra homomorphism. We take any two horizontal vectors $\vec{x}_{1}, \vec{x}_{2}$ of $E$ at the point $x_{0}$. Since the mapping $p: Q \rightarrow E$ is onto, there exist $\vec{g}_{1} \in \hat{\mathbf{G}}, \vec{s}_{1} \in \hat{\mathbf{S}}$ such that $p\left(\vec{g}_{i}+\vec{s}_{i}\right)=\vec{x}_{i}, i=1,2$. The vectors $p\left(\vec{s}_{i}\right)$ are vertical. Hence $p\left(\vec{g}_{i}\right)$ and $\vec{x}_{i}$ have the same horizontal 
component, and hence

$$
2 \Omega\left(\vec{x}_{1}, \vec{x}_{2}\right)=2 \Omega\left(p\left(\vec{g}_{1}\right), p\left(\vec{g}_{2}\right)\right)=\left[\Psi\left(\vec{g}_{1}\right), \Psi\left(\vec{g}_{2}\right)\right]-\Psi\left[\vec{g}_{1}, \vec{g}_{2}\right]=0
$$

The curvature form $\Omega$ vanishes at $x_{0}$ and then must vanish everywhere on account of the homogeneity. The connection $\omega$ is locally flat.

Corollary 2. Let $G$ be a Lie group acting differentiably and transitively on an $n$-dimensional manifold $B$ with $J$ as the isotropic subgroup at a point $b$ of $B$. Denste by $\beta$ the natural linear representation of $J$ on $T_{b}(B)$. Then there is a 1-1 correspondence between invariant linear connections over $B$ and linear mappings $\Psi$ of $\hat{\mathbf{G}}$ into the Lie algebra of $G L(R, n)$ such that

$$
\Psi \circ \operatorname{Ad} . j=\operatorname{Ad} . \beta(j) \circ \Psi, \quad \Psi(\vec{j})=\beta(\vec{j}), \quad j \in J, \quad \vec{j} \in \hat{\mathbf{j}} .
$$

Corollary 3. Let $E, S, G, J, \varphi$ have the same meaning as in Theorem 1. Suppose that $J$ is reductible in $G$ (i.e., $\hat{\mathbf{G}}$ has a linear subspace $\hat{\mathbf{M}}$ such that $\hat{\mathbf{G}}=\hat{\mathbf{J}}+\hat{\mathbf{M}}, \hat{\mathbf{J}} \cap \hat{\mathbf{M}}=0$, Ad. $J(\hat{\mathbf{M}})=\hat{\mathbf{M}})$. Then E always has connections invariant under $G$. Moreover, these connections are in 1-1 correspondence with the linear mappings $\alpha: \hat{\mathbf{M}} \rightarrow \hat{\mathbf{S}}$ such that $\alpha \circ$ (Ad. $j)=\operatorname{Ad} . \varphi(j) \circ \alpha, j \in J$.

Corollaries 2, 3 are particular cases of Theorem 1. Both of them can be regarded as a generalization of a theorem of Nomizu [8]. It is to be noted that the existence of an invariant linear connection does not imply that $J$ is reductible in $G$. A criterion of this reductibility will be given in $\S 9$.

\section{Definition of the group $\Delta$}

To discuss the holonomy group $\Sigma$ of an invariant connection, we find it convenient to introduce a subgroup $\Delta$ of $S$. This group $\Delta$ contains both $\Sigma$ and $\psi(J)$, and is, in general, larger than $\psi(J) \cdot \Sigma$. Elements of $\Delta$ have been used by K. Nomizu [9] to study the affine collineations of Riemannian manifolds. This section and the next are devoted to the discussion of $\Delta$.

Let $\omega$ be a connection over $\{E, S\}$, and $G$ a group of automorphisms of $\{E, S\}$ leaving $\omega$ invariant, not necessarily fibre transitive. From now on, $E$ is assumed to satisfy the second axiom of countability. For each point $x$ of $E$, we denote by $\Sigma_{x}$ the holonomy group at $x$ and by $\mathscr{S}_{x}$ the set of all points which can be joined to $x$ by horizontal curves. These $\mathscr{S}_{2}$ 's are submanifolds of $E$ which we șhall call horizontal manifolds. We note that $\mathfrak{S}_{x}$ is, in general, 
not closed in $E$. Since elements of both $G$ and $S$ carry horizontal curves into horizontal curves, we have

$$
g\left(\mathfrak{S}_{x}\right)=\mathfrak{S}_{g(x)}, \quad s\left(\mathfrak{S}_{x}\right)=\mathfrak{S}_{S(x)}, \quad g \in G, \quad s \in S .
$$

Now fix a point $x$ of $E$. Consider the orbit $\left(\xi_{x}=G\left(\mathfrak{g}_{x}\right)\right.$ of $\mathfrak{F}_{x}$ under $G$. For each $u$ of $\xi_{x}$, let us put

$$
\Delta_{u}=\left\{s: s \in S, s(u) \in \mathbb{E}_{x}\right\} .
$$

We shall see that $\Delta_{u}$ forms a group. In fact, let $s, t \in \Delta_{u}$. There exist $g, h \in G$, $y, z \in \mathfrak{S}_{x}$ such that $s(u)=g(y), t(u)=h(z)$. It follows that

$$
s^{-1} \mathfrak{S}_{x}=s^{-1} \mathfrak{S}_{y}=\mathfrak{H}_{g^{-1}(\mathcal{u})}=g^{-1} \mathfrak{H}_{u} .
$$

Hence

$$
s^{-1} t(u)=s^{-1} h(z)=h s^{-1}(z) \in h s^{-1} \mathfrak{S}_{x}=h g^{-1} \mathfrak{S}_{u} \subset G\left(\mathfrak{S}_{u}\right)=\mathfrak{F}_{x},
$$

and hence $\Delta_{n}$ forms a subgroup of $S$. It is easy to verify that $\Delta_{u}$ is independent of the choice of $u$ in $\xi_{x}$. Nevertheless, if $x$ is changed, $\Delta_{u}$ is changed into one of its conjugate subgroups in $S$.

Let $r \in \Sigma_{u}$, and $s \in A_{u}$. There exist $y \in \mathfrak{S}_{x} . g \in G$ such that $s(u)=g(y)$. Since $r(y) \in \mathfrak{S}_{y}$, we have

$$
s^{-1} r s(u)=s^{-1} r g(y)=g s^{-1} r(y) \in g s^{-1} \mathfrak{S}_{y}=g g^{-1} \mathfrak{S}_{u}=\mathfrak{H}_{u} .
$$

This tells us that $s^{-1} r s \in \mathscr{H}_{u}$. In other words, $\Sigma_{u}$ is a normal subgroup of $\Delta_{u}$. Thus we have proved

(7.1) For each $x$ of $E, \Delta_{x}$ is a subgroup of $S$ and contains $\Sigma_{x}$ as a normal subgroup.

The group $\Delta_{u}$ can also be defined in terms of the parallel displacements. In fact, let $F$ be the fibre containing $u$, and $g$ an element of $G$. Then $g(F)$ is a fibre, and there exists a parallel displacement $\sigma$ (not unique) carrying $g(F)$ to $F$. Let $s=s(g, \sigma)$ be the element in $S$ such that $s(u)=\sigma g(u)$. Then $\Delta_{u}$ consists of the elements $s(g, \sigma)$ for all the possible pairs $(g, \sigma)$.

Since $\Delta_{x}$ is a subgroup of the Lie group $S$, we can talk about its Lie algebra (i.e., the Lie algebra of its arcwise connected component of identity) and its linear adjoint representation. The following property will be found useful :

(7.2) Suppose that $E, S, G, x_{0}, H, p, \omega, \Psi$ have the same meaning as in 
(5.1). If $G$ is transitive on the fibres of $E$, then $\left(\xi_{x_{n}}=G \Delta_{x_{0}}\left(x_{0}\right)\right.$, and $\Psi(\hat{\mathbf{G}})$ is contained in the Lie algebra of $\Delta_{x_{0}}$.

Proof. For simplicity, we write $\left(\xi, 4, \mathfrak{5}, \Sigma\right.$ instead of $\xi_{x_{0}}, \Delta_{x_{0}}, \mathfrak{S}_{x_{0}}, \Sigma_{x_{0}}$. It is evident that $G \Delta\left(x_{0}\right) \subset G(\mathfrak{S})=$ (5. To see the equality, let $y \in \mathfrak{F}$. There exist $z \in \mathfrak{H}, g \in G$ such that $g(z)=y$. Since $G$ is fibre-transitive, we can find $g_{1} \in G$ such that $x_{0}$ and $g_{1}^{-1} g(z)$ are in the same fibre. There exists then $s \in S$ with the property that $s\left(x_{0}\right)=g_{1}^{-1} g(x) \in G(\mathfrak{S})=(\mathfrak{5}$. By definition of $\Delta, s \in \Delta$. Therefore $y=g(z)=g_{1} s\left(x_{0}\right) \in G \Delta\left(x_{0}\right)$. This shows the equality $G \Delta\left(x_{0}\right)=E$.

Since $\Delta$ is a subgroup of the Lie group $S$, we can give it a stronger topological structure to render it a Lie group $\Delta^{\prime}$ in such a manner that $\Delta$ and $\Delta^{\prime}$ have the same arcwise connected components. Let us consider the Lie group $G \times \Delta^{\prime}$. It contains $H$ as a closed subgroup. Denote by $(5)$ the coset space $\left(G \times \Delta^{\prime}\right) / H$. There is a $1-1$ continuous mapping $i$ of $\left(\xi^{\prime}\right.$ onto $(\xi \%$ In the natural manner, $\left\{\left[^{\prime}, \Delta^{\prime}\right\}\right.$ forms a differentiable principal fibre space. We can readily verify that $\left\{E^{\prime}, \Delta^{\prime}\right\}$ together with $i$ is a reduced bundle of $\{E, S\}$ in the sense of Nomizu [10, p. 37]. Thus both $\left[\right.$ and $\Delta\left(x_{0}\right)$ are submanifolds of $E$.

Since $\mathscr{S} \subset \mathbb{E}$, the connection $\omega$ on $\{E, S\}$ can be reduced to a connection on $\left\{\xi^{\prime}, \Delta^{\prime}\right\}[10$, p. 27]. In other words, any horizontal vector at a point of $\mathbb{E}$ is tangent to $F$. Let $\vec{g} \in \hat{\mathbf{G}}$, then $p(\vec{g}) \in T_{x_{0}}(\mathfrak{F})$. From the definition of $\Psi$ (cf. $\S 5$ ), the horizontal and vertical components of $p(\vec{g})$ are, respectively, $p(\vec{g}+\Psi(\vec{g}))$ and $-p(\Psi(\vec{g}))$. Since $p(\vec{g}+\Psi(\vec{g})) \in T_{x_{0}}(E)$, we know that $p \Psi(\vec{g}) \in T_{x_{0}}(E)$. The vector $p \Psi(\vec{g})$, being vertical, then must be tangent to the orbit $\Delta\left(x_{0}\right)$ of $x_{0}$ under $\Delta$. There exists $\vec{s} \in \hat{s}$ such that $p(\vec{s})=p \Psi(\vec{g})$. We know that, restricted to $\hat{\mathbf{s}}, p$ is one to one. Hence $\Psi(\vec{s})=\vec{s} \in \hat{\jmath}$. The proof is thus completed.

Professor Nomizu has communicated to me that $\Psi(\hat{\mathbf{G}}) \subset \hat{\Delta}$ still holds if $G$ is not fibre-transitive.

\section{Some remarks about the group $\Delta$}

We shall give, in this section, three remarks about $\Delta$ which will not be used in the rest of this paper.

Remark 1. Let $N$ be the normaliser of $\Sigma_{x}$ in $S$, and

$$
\mathfrak{M}=N\left(\mathfrak{S}_{x}\right)=\left\{n(y): n \in N, y \in \mathfrak{S}_{x}\right\} .
$$


Then $\mathfrak{M}$ is a closed submanifold of $E$, and $\{\mathfrak{M}, N\}$ together with the identity mapping forms a reduced bundle of $\{E, S\}$. For each $y \in \mathfrak{M}, \mathfrak{F}_{y} \subset \mathfrak{M}$. Since any two horizontal manifolds are either disjoint or identical, we can decompose $\mathfrak{M}$ by horizontal manifolds. Let $\bar{M}$ denote the decomposition space. This space may not be Hausdorff as the holonomy group $\Sigma_{x}$ may not be closed in $N$. The group $N$ acts on $\bar{M}$ in the natural manner as a transitive topological transformation group. Let $\bar{x} \in \bar{M}$ be the image of $x$ under the decomposition mapping. Then the isotropic subgroup of $N$ at $\bar{x}$ is the holonomy group $\Sigma_{x}$ at $x$ (in fact, $\Sigma_{x}$ leaves every point of $\bar{M}$ fixed). There is a 1-1 continuous mapping $\alpha$ of the factor group $N / \Sigma_{x}$ onto $\bar{M}$. We note that $N / \Sigma_{x}$ is, in general, non-Hausdorff. The mapping $\alpha$ is, moreover, a homeomorphism. This follows from the fact that, given any open subset $U$ of $S$, the set

$$
U\left(\mathfrak{S}_{x}\right)=\left\{u(y): u \in U, y \in \mathfrak{H}_{x}\right\}
$$

is open in $E$.

On the other hand, we have the group $G$ of automorphisms of $\{E, S, \omega\}$ (not necessarily fibre-transitive). Since $\Delta_{x} \subset N, G$ carries $\mathfrak{M}$ into itself. Each $g$ of $G$ permutes the horizontal manifolds Thus $G$ acts on the decomposition space $\overline{\mathfrak{M}}$ in the natural manner as a topological transformation group. Define $\beta: C \rightarrow N / \Sigma_{x}$ by $\beta(g)=\alpha^{-1} g(\bar{x}), g \in G$. Then $\beta$ is continuous. From the commutativity of elements of $G$ and elements of $S$, we know that $\beta$ is a homomorphism. Moreover, $\beta(G)=\Delta_{x} / \Sigma_{x}$. In other words,

The group $\Delta_{x}$ is an extension of the holonomy group $\Sigma_{x}$ by a homomorphic image $\beta(G)$ of $G$.

An example: Let $B_{n}$ be the $n$-dimensional ordinary affine space deprived of the origin, and $G$ the group of all affine collineation of $B_{n}$. Since the connection is flat, the holonomy group contains only the identity. Both $G$ and $\Delta$ are isomorphic with the general real linear group $G L(R, n)$, and $\beta$ is an isomorphism. The isotropic subgroup $J$ is of dimension $n^{2}-n$.

Remark 2. Suppose that $\{E, S\}$ is the bundle of frames of a differentiable manifold $B$. Then $\omega$ is the linear connection over $B$. From the second definition of $\Delta$ and the fact that $\Delta_{x} \subset N$, we have the following: ${ }^{3)}$

3) This has been proved in [5]. 
Let $T$ be a parallel tensor field over $B$. If at a point $b$ of $B, T_{b}$ is invariant under (the connected component of identity of) the normalizer of the holonomy sroup at $b$, then $T$ is invariant under (the connected component of identity of) the group of all affine collineations of $B$.

Remark 3. Let $\{E, S\}$ be a differentiable principal fibre bundle with a connection. It is well-known that the holonomy group can be regarded as either a subgroup of the structure group $S$ or a group of automorphisms of a fibre $F$ which commutes with $S$ elementwise. Used in the former sense, it depends on a point $x$ of reference, and we shall denote it by $\Sigma_{x}$. Used in the latter sense, it depends on the fibre $F$, and we shall denote it by $\Sigma_{F}$. The situation is similar for the group $\Delta$. In the above discussions, we fix a point $x$ of $E$, and consider $\Delta_{x}$ as a subgroup of $S$. If we use the second definition of $\Delta$, we can also interprete it as a group of automorphisms of $F$ which commute elementwise with $S$. Thus $\Delta$ depends on $F$. When used in this sense, it will be denoted by $A_{F}$. For each $\left(\xi_{x}=G\left(\mathscr{F}_{x}\right)\right.$ and each fibre $F$, the intersection $F_{x} \cap F$ is an orbit of $\Delta_{F}$. Here we note that infinitesimal trnasformations ${ }^{1)}$ of $\Delta_{F}$ have a meaning. They are vector fields over $F$ and are invariant under the translations of the structure group $S$.

If $G$ is connected, then the following four conditions are equivalent: (a) $G$ leaves invariant the horizontal manifold $\mathfrak{H}_{x}$; (b) $\Sigma_{x}=\Delta_{x}$; (c) at each point of $\mathfrak{S}_{x}$, every infinitesimal transformation of $G$ is tangent to $\mathscr{S}_{x}$; (d) on any fibre $F$ of $E$, the field of vertical components of each infinitesimal transformation of $G$ is an infinitesimal transformation of $\Sigma_{F}$.

Proof. The equivalence of (a) and (b) is evident while the equivalence of (a) and (c) follows from the fact that $G$ is generated by its infinitesimal transformations. To discuss condition (d), let $Z$ be an infinitesimal transformation of $G$, and denote by $Z_{v}$ the field of vertical components of $Z$. Since $G$ and $S$ commute elementwise, the vector field $Z$, and hence $Z_{v}$, is invarant under $S$. Choose a fibre $F$, a point $y$ on $F$, and denote by $Z(y), Z_{v}(y)$, respectively, the values of $Z, Z_{v}$ at $y$. Suppose that (c) holds. Then $Z(y) \in T_{y}\left(\xi_{y}\right)$. Since

\footnotetext{
4) Vector fields and infinitesimal transformations on a differentiable manifold are synonymous. By an infinltesimal transformation of a differentiable transformation group, we mean the infinitesimal transformation induced by a one-parameter subgroup of that transformation group.
} 
$T_{y}\left(\mathfrak{S}_{y}\right)$ contains all the horizontal vectors of $E$ at $y$, we have

$$
Z_{v}(y) \in T_{y}\left(\xi_{2}\right) \cap T_{y}(F)=T_{y}\left(\xi_{y} \cap \cap F\right) .
$$

There exists then an infinitesimal trnasformation $W$ of $\Sigma_{F}$ such that $Z_{v}(y)$ $=W(y)$. Both vector fields $Z_{v}$ and $W$ are invariant under $S$, and $S$ is transitive on $F$. It follows that $Z_{v}$ and $W$ coincide at every point of $F$. In other words, condition (d) holds. Conversely, suppose that (d) holds. Then $Z_{v}$ is tangent to horizontal manifolds $\mathfrak{S}_{y}$ at every point $y$ of $E$. Since $T_{y}\left(\mathfrak{S}_{y}\right)$ contains all the horizontal vectors at $y$, we have $Z(y) \in T_{y}\left(\mathfrak{S}_{y}\right)$. This completes the proof.

Remark 4. Let $B$ be a differentiable manifold and $E$ the bundle of frames over $E$. Choose a point $b$ of $B$, and denote by $F$ the corresponding fibre in $E$. Since $E$ is the principal fibre bundle associated with the tangent bundle of $B$, there is a one-to-one correspondence between automorphisms of $T_{b}(B)$ and automorphisms of $F$ commuting with all elements of $S$. Passing to the infinitesimal transformations, we have a correspondence $L$ between endomorphisms of $T_{b}(B)$ and vector fields over $F$ invariant under $S$. This correspondence $L$ is bijective, linear and preserve the bracket operation. $L$ also gives a correspondence ${ }^{5)}$ between tensor fields of type $(1,1)$ on $B$ and vertical vector fields on $E$ which are invariant under $S$.

Now suppose that $B$ has a linear connection without torsion. For each vector field $X$ on $B$, Kostant [12] defined a field $a_{X}$ of endomorphisms of the tangent spaces of $B$ by putting $a_{X}(v)=-\nabla_{v} X, v \in T(B)$, where $\nabla$ denotes the covariant differentiation. This field $a_{X}$ has a very simple geometrical meaning in the bundle $E$ of frames. In fact, $X$, being an infinitesimal transformation on $B$, gives in the natural manner an infinitesimal transformation $\widetilde{X}$ on the bundle $E$ of frames. Let $\tilde{X}_{v}$ be the field of vertical components of $\widetilde{X}$. By a direct computation, we can verify that $L\left(a_{X}\right)=\widetilde{X}_{v}$. By means of the correspondence $L$, both the group $\Delta_{F}$ and the holonomy group $\Sigma_{F}$ can be regarded as groups of automorphisms of the tangent space. Thus we have, from Remark 3,

Let $G$ be a connected group of affine collineations of a manifold $B$ with a

5) Let $u^{i}(i=1,2, \ldots, n)$ be a system of local coordinates of $B$, and $\left(u^{i}, X_{j}^{i}\right)$ be the local coordinates in $E$ as in [10, Chap. III, $\$ 4]$. Suppose A to be a tensor field of type $(1,1)$ on $B$ defined by $A\left(\partial / \partial u^{j}\right)=A_{i}^{k} \partial / \partial u^{k}$, where $A_{i}^{k}$ are functions of $u^{\prime}$ s. Then $L(A)$ is the vector field $-X_{j}^{\prime} A_{i}^{k} \partial / \partial X_{j}^{k}$ on $E$. 
linear connection. Then the group 4 coinsides with the holonomy group if and only if, for eash infinitesimal transformation $X$ of $G$, the Kostant tensor $a_{x}$ belongs to the Lie algebra of the holonomy group.

Kostant [12] has proved that, for and Killing vector field $X$ on a compact Riemannian manifold, $a_{x}$ always belongs to the Lie algebra of the holonomy group. Hence

Let $G$ be a connected group of isometries of a compact Riemannian space $B$. Then the group $\Delta$ and the holonomy group coincide. In particular, at each point of $B$, the linear isotropic subgroup is contained in the holonomy group. Moreover, each parallel tensor field over $B$ is invariant under $G$.

If $B$ is, moreover, homogeneous, this has been proved by Kostant (to appear). For $B$ irreducible, this can also be obtained from Lichnerowicz results [6].

\section{The holonomy group}

Now assume that $G$ is fibre-transitive on $E$. We have shown in $\S 6$ that the invariant connections $\omega$ are in 1-1 correspondence with certain linear mappings $\Psi: \hat{\mathbf{G}} \rightarrow \hat{\mathbf{S}}$. It is the aim of this section to give an explicit expression of $\Delta$ and the holonomy group $\Sigma$ in terms of $\Psi$. When $J$ is reductible in $G$ and $\omega$ is the canonical linear connection of the second kind, the expression for $\Sigma$ has been found by K. Nomizu.

THEOREM 2. Let $G$ be a Lie group of automorphisms of $\{E, S\}$, transitive on the fibres. Suppose that $\omega$ is an invariant connection corresponding to the linear mapping $\Psi: \hat{\mathbf{G}} \rightarrow \hat{\mathbf{S}}$. Denote by $\hat{\mathbf{R}}$ the subalgebra of $\hat{\mathbf{S}}$ generated by $\Psi(\hat{\mathbf{G}})$. Then $\Delta_{x_{0}}=\psi(J) \cdot R$ where $R=\exp . \hat{\mathbf{R}}$. If, moreover, $\psi(J)$ is arcwise connected, then $\Delta_{x_{0}}=R$.

Proof. Since $\Psi \circ \operatorname{Ad} .(j)=\operatorname{Ad} . \varphi(j) \circ \Psi$ for $j \in J$, we have Ad. $\varphi(J)(\hat{\mathbf{R}})=\hat{\mathbf{R}}$ and Ad. $\psi(J)(R)=R$. In other words, $\psi(J)$ belongs to the normalizer of $R$, and hence $\psi(J) \cdot R=R \cdot \psi(J)$ forms a subgroup of S. From (7.2) and the definition of $\Delta$, we have $R \subset \Delta_{x_{0}}$ and $\phi(J) \subset \Delta_{x_{0}}$ respectively. Therefore $\phi(J) \cdot R \subset \Delta_{x_{0}}$.

To prove $\Delta_{x_{0}} \subset \psi(J) \cdot R$, we shall first show that the connection $\omega$ can be reduced to a connection with structure group $\psi(J) \cdot R$, and then show the required inclusion, For this purpose, we give the subgroup $\psi(J) \cdot R$ of $S$ its 
intrinsic topology to render it a Eie group $\Gamma$. Let $\left(5=G R\left(x_{0}\right)\right.$ and $(5)=.(G \times \Gamma) / H$ where $H=\{(j, \psi(j)): j \in J\}$. The mapping $i: C^{\prime} \rightarrow E$ defined by $i((g, s) H)$ $=g s\left(x_{0}\right), g \in G, s \in \Gamma$, is one-to-one and differentiable. Moreover, $i\left(\varsigma^{\prime}\right)=(\varsigma$. Assuming $\Gamma$ to act on (S!' from the left in the natural manner, we can readily verify that $\{\S \prime, \Gamma\}$ together with $i$ is a reduced bundle of $\{E, S\}$ in the sense of $[10$, p. 37]. Now we shall see that the connection $\omega$ can be reduced to a connection on $\left\{C^{\prime}, \Gamma\right\}$. Let $y \in\left(5=G R\left(x_{0}\right)\right.$, and $\vec{y}$ be a horizontal vector at $y$. There exist $g \in G, r \in R$, such that $g r(y)=x_{0}$. Then $\vec{x}=\operatorname{gr}(\vec{y}) \in T_{x_{0}}(E)$ and is horizontal. Since $Q=G \times S$ acts transitively on $E$, we can find $\vec{g} \in \hat{\mathbf{G}}, \vec{s} \in \hat{\mathbf{S}}$ with the property that $p(\vec{g}+\vec{s})=\vec{x}$. From the equality

$$
0=\omega(x)=\left(p^{*} \omega\right)(\vec{g}+\vec{s})=s-\Psi(\vec{g})
$$

it follows that $\vec{s}=\Psi(\vec{g})$, and then $\vec{x}=p(\vec{g}+\Psi(\vec{g})) \in p(\hat{\mathbf{G}}+\hat{\mathbf{R}})=T_{x_{0}}(C)$. Therefore, $\vec{y}=r^{-1} g^{-1}(\vec{x}) \in T_{y}(\S)$ because $R G(\S)=\S$. In other words, $\omega$ can be reduced to a connection on $\left\{\S^{\prime}, \Gamma\right\}$. We have then

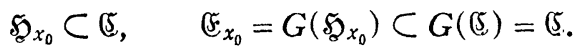

As $\Delta^{\prime}$ and $\Gamma$ are the structure groups of $\left(S^{\prime}\right.$ and $\left({ }^{\prime} \prime\right.$ respectively, it follows that $\Delta_{x_{0}} \subset \psi(J) \cdot R$. The equality $\Delta_{x_{0}}=\psi(J) \cdot R$ is thus established.

Since $\psi(\hat{\mathbf{J}})=\Psi(\hat{\mathbf{J}}) \subset \Psi(\hat{\mathbf{G}}) \subset \hat{\mathbf{R}}$, we have $\psi(J) \subset R$ and $\Delta_{x_{0}}=R$ whenever $\phi(J)$ is arcwise connected.

TheOREM 3. Assumptions are the same as in Theorem 2. Let $V$ denote the linear subspace of $\hat{\mathbf{S}}$ spanned by $\left\{\Psi\left[\vec{g}_{1}, \vec{g}_{2}\right]-\left[\Psi\left(\vec{g}_{1}\right), \Psi\left(\vec{g}_{2}\right)\right]: \vec{g}_{1}, \vec{g}_{2} \in \hat{\mathbf{G}}\right\}$. Then the Lie algebra $\hat{\Sigma}$ of the holonomy group $\Sigma$ at $x_{0}$ is the minimal linear subspace containing $\hat{\mathrm{V}}$ and invariant under Ad. $\Delta_{x_{0}}$, or what is the same,

$$
\hat{\mathbf{V}}+[\Psi(\hat{\mathbf{G}}), \hat{\mathbf{V}}]+[\Psi(\hat{\mathbf{G}}),[\Psi(\hat{\mathbf{G}}), \hat{\mathbf{V}}]]+\ldots .
$$

Proof. Let $\Omega$ denote the curvature form of the connection $\omega$, and $\Omega=p^{*} \Omega$. From Theorem 1,

$$
2 \Omega\left(\vec{g}_{1}, \vec{g}_{2}\right)=\left[\Psi\left(\vec{g}_{1}\right), \Psi\left(\vec{g}_{2}\right)\right]-\Psi\left[\vec{g}_{1}, \vec{g}_{2}\right], \quad \vec{g}_{1}, \vec{g}_{2} \in \hat{\mathbf{G}} .
$$

Hence $\hat{\mathrm{v}} \subset\left\{\Omega\left(\vec{x}_{1}, \vec{x}_{2}\right): \vec{x}_{1}, \vec{x}_{2} \in T_{x_{0}}(E)\right\}$. We shall see that they actually coincide. Given $\vec{x}_{i} \in T_{x_{0}}(E)$, there exist $\vec{g}_{i} \in \hat{\mathbf{G}}, \overrightarrow{\boldsymbol{s}}_{i} \in \hat{\mathbf{S}}$ such that $p\left(\vec{g}_{i}+\vec{s}_{i}\right)$ $=\vec{x}_{i}, i=1,2$. The vectors $p\left(\vec{g}_{i}\right)$ and $\vec{x}_{i}$ have the same horizontal component. Hence $\Omega\left(\vec{g}_{1}, \vec{g}_{2}\right)=\Omega\left(p\left(\vec{g}_{1}\right), p\left(\vec{g}_{2}\right)\right)=\Omega\left(\vec{x}_{1}, \vec{x}_{2}\right)$, and hence 


$$
\hat{\mathrm{v}}=\left\{\Omega\left(\vec{x}_{1}, \vec{x}_{2}\right): \vec{x}_{1}, \vec{x}_{2} \in T_{x_{0}}(E)\right\}
$$

Let $\hat{\mathbf{U}}$ be the minimal linear subspace of $\hat{\mathbf{S}}$ containing $\hat{\mathbf{V}}$ and invariant under Ad. $\Delta_{x_{0}}$. Since $\hat{\mathrm{V}} \subset \hat{\Sigma}$, proposition (7.1) implies that $\hat{\mathbf{U}} \subset \hat{\Sigma}$. We note here that $\hat{\mathbf{U}}$ is an ideal of the Lie algebra of $\Delta_{x_{0}}$ and hence a subalgebra. Let $y$ be any point on $\mathfrak{S}_{x_{0}}$ and $\vec{y}_{1}, \vec{y}_{2} \in T_{y}(E)$. Since $\mathfrak{S}_{x_{0}} \subset \mathfrak{F}_{x_{0}}$ and $G \times \Delta_{x_{0}}$ is transitive on $\mathfrak{F}_{x_{0}}$, there exist $g \in G, s \in \Delta_{x_{0}}$ such that $g s(y)=x_{0}$. Hence $g s\left(\vec{y}_{i}\right)$ $\in T_{x_{0}}(E)$, and we have

$$
\Omega\left(\vec{y}_{1}, \vec{y}_{2}\right)=\Omega\left(g\left(\vec{y}_{1}\right), g\left(\vec{y}_{2}\right)\right)=\left(\text { Ad. } s^{-1}\right) \Omega\left(g s\left(\vec{y}_{1}\right), g s\left(\vec{y}_{2}\right)\right) \in \text { Ad. } \Delta_{x_{0}}(\hat{\mathbf{v}}) \subset \hat{\mathbf{U}} \text {. }
$$

A theorem of Ambrose-Singer [1] then tells us that $\hat{\Sigma} \subset \hat{\mathbf{U}}$. Hence $\hat{\mathbf{U}}=\hat{\Sigma}$.

Let $\hat{\mathbf{W}}=\hat{\mathbf{V}}+[\Psi(\hat{\mathbf{G}}), \hat{\mathbf{V}}]+[\Psi(\hat{\mathbf{G}}),[\Psi(\hat{\mathbf{G}}), \hat{\mathbf{V}}]]+\ldots$ Both $\operatorname{Ad} . \psi(J)$ and Ad. $R$ leaves $\hat{\mathbf{W}}$ invariant. The former follows from the formula $\Psi$. Ad. $j$ $=\operatorname{Ad} . \psi(j) \circ \Psi, j \in J$, while the latter from the fact that $\hat{\mathbf{R}}$ is generated by $\Psi(\hat{\mathbf{G}})$ and $R=\exp . \hat{\mathbf{R}}$. Therefore, $\hat{\mathbf{U}} \subset \hat{\mathbf{W}}$. The inclusion $\hat{\mathbf{W}} \subset \hat{\mathbf{U}}$ is evident and we get $\hat{\mathbf{U}}=\hat{\mathbf{W}}$. This completes the proof of Theorem 3 .

Corollary. If, in Theorem $3, \Psi(\hat{\mathbf{G}})=\psi(\hat{\mathbf{J}})$, then the Lie algebra of the holonomy group at $x_{0}$ is the linear space $\hat{\mathbf{v}}$.

Proof. This follows from the fact that $[\Psi(\hat{\mathbf{G}}), \hat{\mathbf{V}}]=[\psi(\hat{\mathbf{J}}), \hat{\mathbf{V}}] \subset \hat{\mathbf{V}}$.

Remark. Let $\{E, S\}$ be the bundle of frames of a manifold $B$, and assume that $G$ to be effective. Then the homomorphism $\psi: J \rightarrow S$ is an isomorphism. Suppose that $E$ has an invariant connection for which $\Psi(\hat{\mathbf{G}})=\psi(\hat{\mathbf{J}})$. Denote by $\hat{\mathbf{K}}$ the kernel of $\Psi$. Then $\hat{\mathbf{K}} \cap \hat{\mathbf{J}}=0$, and $\operatorname{dim} . \hat{\mathbf{K}}=\operatorname{dim} . \hat{\mathbf{G}}-\operatorname{dim} . \psi(\hat{\mathbf{J}})$ $=\operatorname{dim} . \hat{\mathbf{G}}-\operatorname{dim} . \hat{\mathbf{J}}$. Hence $\hat{\mathbf{G}}=\hat{\mathbf{K}}+\hat{\mathbf{J}} . \quad$ From the fact $\Psi \circ \operatorname{Ad} . j=\operatorname{Ad} . \psi(j) \circ \Psi$, $j \in J$, it follows that $(\operatorname{Ad} . J)(\hat{\mathbf{K}})=\hat{\mathbf{K}}$. This tells us that $J$ is reductible in $G$. Conversely, suppose that $J$ is reductible in $G$. Let $\hat{\mathbf{G}}=\hat{\mathbf{J}}+\hat{\mathbf{K}}$ be the reduction. We define the linear mapping $\Psi: \hat{\mathbf{G}} \rightarrow \hat{\mathbf{S}}$ by putting $\Psi(\vec{j})=\psi(\vec{j}), \Psi(\vec{k})=0$, $\vec{j} \in \hat{\mathbf{J}}, \vec{k} \in \hat{\mathbf{K}}$. This $\Psi$ gives an invariant connection with the property $\Psi(\hat{\mathbf{G}})$ $=\psi(\hat{\mathbf{J}})$. It can be verified that this is Nomizu's canonical connection of the second kind [8].

\section{Invariant Cartan connection}

Let $\{E, S\}$ be a differentiable principal fibre space with base space $B$, and $S_{1}$ a Lie group containing $S$ such that $\operatorname{dim} . B=\operatorname{dim} . S_{1} / S$. Denote by $\hat{\mathbf{S}}, \hat{\mathbf{s}}_{1}$ the Lie algebras of $S, S_{1}$ respectively. Then a Cartan connection over $B$ can be defined 
as an $\hat{\mathbf{S}}_{1}$-valued linear differential from $\omega$ over $E$ with the following properties:

(i) $\vec{x} \in T(E)$ and $\omega(\vec{x})=0$ implies $\vec{x}=0$;

(ii) $s^{*} \omega=($ Ad. $s) \omega, s \in S$;

(iii) For any $x \in E$, let us define $\pi_{x}: S \rightarrow E$ by $\pi_{x}(s)=s(x), s \in S$. Then $\pi_{x}^{*} \omega$ is the right Maurer-Cartan form of $S$ [3], [4].

Using the same arguments as before, we can prove

Theorem 4. Suppose $G$ to be a Lie group of automorphisms of $\{E, S\}$, transitive on the fibres. Then there is a 1-1 correspondence between the in. variant Cartan connections $\omega$ over $B$ and linear mappings $\Psi: \hat{\mathbf{G}} \rightarrow \hat{\mathbf{S}}_{1}$ such that (a) $\Psi(\vec{j})=\psi(\vec{j}), \vec{j} \in \hat{\mathbf{J}}$, (b) $\Psi \circ$ Ad. $j=$ Ad. $\psi(j) \circ \Psi, j \in J$, and (c) $\Psi(\hat{\mathbf{G}})+\hat{\mathbf{s}}$ $=\hat{\mathbf{S}}_{1}$. Here, $J, \hat{\mathbf{J}}, \psi$ have the same meaning as in $\S 4$.

\section{ReFERENCES}

[1] W. Ambrose and I. Singer, A theorem on holonomy, Trans. Amer. Math. Soc., 75 (1953), 428-433.

[2] S. S. Chern, Topics in differential geometry, Institute for Advanced Study notes, 1951.

[3] C. Ehresmann, Les connexions infinitésimal dans un espaces fibré differentiable, Coll. de topologie, Bruxelles, 1950, 29-55.

[4] S. Kobayashi, On connections of Cartan, Canadian J. of Math., 8 (1956), 145-156.

[5] S. Kobayashi and K. Nomizu, On automorphisms of a Kählerian structure, Nagoya Math. J., 11 (1957), 115-124.

[6] A. Lichnerowicz, Espaces homogènes Kählériens, Coll. géo. diff., Strasbourg, 1953, 171-184.

[7] D. Montgomery and L. Zippin, Topological transformation groups, New York, 1955.

[8] K. Nomizu, Invariant affine connections on homogeneous spaces, Amer. J. of Math, 76 (1954), 33-65.

[9] K. Nomizu, Studies on Riemannian homogeneous spaces, Nagoya Math. J., 9 (1955), 43-56.

[10] K. Nomizu, Lie groups and differential geometry, Math. Soc. of Japan, 1956.

[11] K. Yano, On harmonic and Killing vectors, Ann. of Math., 55 (1952), 38-45.

[12] B. Kostant, Holonomy and the Lie algebra of infinitesimal motions of a Riemannian manifold, Trans. Amer. Math. Soc., 80 (1955), 528-542.

[13] B. Kostant, On holonomy and homogeneous spaces, Nagoya Math. J. (To appear).

\section{Northwestern University}

